\title{
NOMBRE DE CAS CONFIRMÉS DE SYPHILIS CHEZ LES FEMMES ENCEINTES AU BRÉSIL ENTRE 2009 ET 2013
}

\section{ARTICLE ORIGINAL}

BARROS, Yara Lorrane Souza de ${ }^{1}$, FECURY, Amanda Alves², OLIVEIRA, Euzébio de $^{3}$, DENDASCK, Carla Viana ${ }^{4}$, ARAÚJO, Maria Helena Mendonça de ${ }^{5}$, SOUZA, Keulle Oliveira $\mathrm{da}^{6}$, DIAS, Claudio Alberto Gellis de Mattos $^{7}$

BARROS, Yara Lorrane Souza de. Et al. Nombre de cas confirmés de syphilis chez les femmes enceintes au Brésil entre 2009 et 2013. Revista Científica Multidisciplinar Núcleo do Conhecimento. An 05, Ed. 11, vol. 25, p. 53-61. novembre 2020. ISSN: 2448-0959,

Lien d'accès:

https://www.nucleodoconhecimento.com.br/sante/femmes-enceintes, DOI: 10.32749/nucleodoconhecimento.com.br/sante/femmes-enceintes

\section{RÉSUMÉ}

La syphilis est une maladie bactérienne transmise sexuellement des femmes enceintes atteintes de syphilis au fœtus. Le diagnostic de syphilis chez les femmes enceintes est confirmé par l'examen VDRL. L'objectif de ces travaux est de montrer le nombre de cas confirmés de syphilis chez les femmes enceintes au Brésil, comme une année de diagnostic, groupe d'âge, régions du Brésil, zone résidentielle, type de test, classification et évolution entre 2009 et 2013. Des données secondaires ont été utilisées par le département informatique de SUS - DATASUS

\footnotetext{
${ }^{1}$ Technique minière, à la suite de l'Institut fédéral d'Amapá (IFAP).

${ }^{2}$ Biomédical, Docteur en maladies tropicales, Professeur et chercheur du Cours médical de l'Université fédérale d'Amapá (UNIFAP).

${ }^{3}$ Biologiste, PhD en maladies topiques, professeur et chercheur du cours d'éducation physique de l'Université fédérale du Pará (UFPA).

${ }^{4}$ Théologien, Docteur en psychanalyse, chercheur au Center for Research and Advanced Studies - CEPA.

${ }^{5}$ Médecin, professeur et chercheur du cours de médecine de l'Université fédérale d'Amapá (UNIFAP).

${ }^{6}$ Sociologue, étudiant à la maîtrise en études anthropiques en Amazonie, membre du groupe de recherche «Laboratoire d'éducation, d'environnement et de santé » (LEMAS/UFPA).

${ }^{7}$ Biologiste, $\mathrm{PhD}$ en théorie et recherche comportementale, professeur et chercheur du Graduate Program in Professional and Technological Education (PROFEPT), Federal Institute of Amapá (IFAP).
}

RC: 68387

Disponible en: https://www.nucleodoconhecimento.com.br/sante/femmes-enceintes 
(http://datasus.saude.gov.br). Le plus grand nombre de tests permet un meilleur traitement et une réduction du nombre de cas. Cependant, l'absence de soins préventifs pendant la relation sexuelle induit une augmentation des cas, en particulier chez les femmes en âge de procréer. Les régions où la population est plus importante ont un plus grand nombre de cas. La forte variation des partenaires dans ces domaines entraîne une contamination accrue. Le type de test influence les nombres. Des tests moins spécifiques et plus généraux trouvent des anticorps spécifiques et non spécifiques pour la bactérie. Cela peut modifier les nombres réels. Les soins de santé féminins font à la fois le diagnostic tôt, facilitant le traitement. La syphilis n'est pas directement responsable du nombre plus élevé de décès chez les femmes enceintes.

Mots-clés: Épidémiologie, syphilis, femmes enceintes.

\section{INTRODUCTION}

La syphilis est une maladie bactérienne qui touche l'ensemble du corps, transmise sexuellement; par don de sang; contact direct avec du sang contaminé; et la femme enceinte avec la syphilis pour le foetus. La bactérie pathogène est Treponema Pallidum (CARVALHO et coll., 2014, JUNIOR et al., 2009; ROCHA et al., 2020).

Les symptômes de la syphilis se manifestent en trois phases. Dans la première phase peut apparaître des taches rouges sur le corps, les blessures généralement sur les organes génitaux. Dans la deuxième phase, il est possible que la maladie soit dormante pendant une période de huit mois, lorsque la bactérie peut être distribuée affectant la peau et les organes. Dans la troisième phase, il est courant d'apparaître des inflammations dans les tissus du corps, tels que les os, les muscles et le foie (AVELLEIRA et coll., 2006; BRETAS et al., 2008).

La maladie doit être diagnostiquée afin que le patient puisse faire le traitement approprié, en empêchant la transmission de la maladie par l'utilisation de préservatifs masculins/féminins. Le traitement le plus efficace est la pénicilline de benzaycine de

RC: 68387

Disponible en: https://www.nucleodoconhecimento.com.br/sante/femmes-enceintes 
drogue. Jusqu'en 2015, il n'y avait aucune trace de cas de résistance à celui-ci. D'autres médicaments sont utilisés dans le traitement de la syphilis, mais celui qui a le plus d'efficacité dans le traitement/guérison est ce type de pénicilline (KALININ et coll., 2015; BRASIL, 2015).

Le diagnostic de syphilis chez les femmes enceintes est confirmé par l'examen VDRL (Venereal Disease Research Laboratory) pendant les soins prénatals. La découverte de la maladie et le traitement des femmes enceintes pendant les soins prénatals à la pénicilline préviennent la syphilis congénitale (SUTO et coll., 2016; MESQUITA et al., 2012).

Le nombre de cas de syphilis chez les femmes enceintes au Brésil était de 21382 en 2012 et 2013 (BRASIL 2015).

Le nombre de cas de syphilis chez les femmes enceintes dans la région du Nord-Est était de 4433 en 2012 et 2013. Au cours de la même période, le nombre de cas de syphilis chez les femmes enceintes dans la région du Sud-Est était de 10 052; dans la région du Sud était 2795; dans la région du Midwest était en 1961; et dans la région nord, il était de 214 (BRASIL, 2015).

Le nombre de cas de syphilis chez les femmes enceintes à Amapá était de 176 en 2012 et 2013 (BRASIL 2015).

\section{BUT}

L'objectif de cet article est de montrer le nombre de cas confirmés de syphilis chez les femmes enceintes au Brésil, tels que l'année du diagnostic, le groupe d'âge, les régions du Brésil, la zone résidentielle, le type de test, la classification et l'évolution entre 2009 et 2013.

\section{MÉTHODE}

RC: 68387

Disponible en: https://www.nucleodoconhecimento.com.br/sante/femmes-enceintes 
Données prises auprès du service informatique de SUS - DATASUS (http://datasus.saude.gov.br) suivant les étapes suivantes : premièrement, l'onglet « accès à l'information » a été sélectionné, suivi de l'option " information sur la santé (TABNET) » peu après l'option "épidémiologique et morbidité ॥. II a cliqué sur l'option « Maladies et maladies à déclaration obligatoire à partir de 2007 (SINAN) 》 puis «Syphilis chez les femmes enceintes", et dans l'onglet « couverture géographique » sélectionnée «Brésil par région, UF et municipalité ». Les étapes suivantes ont été: A) dans la rangée sélectionné «Cas confirmés selon l'année du diagnostic », dans la colonne «pas actif » et dans le contenu « cas confirmés». Pour cette option et pour les données suivantes ont été recueillies de 2009 à 2013. B) dans la ligne, les « cas confirmés selon la région de notification » ont été sélectionnés, dans la colonne « non actif » et dans le contenu des « cas confirmés». C) dans la rangée, les « cas confirmés selon le groupe d'âge » ont été sélectionnés, dans la colonne « non actif » et dans le contenu des « cas confirmés ». D) dans la ligne, «Cas confirmés selon Evolution », dans la colonne « non actif » et dans le contenu « cas confirmés » ont été sélectionnés. E) dans la rangée, des « cas confirmés selon la classification clinique », dans la colonne « non actif » et dans le contenu « cas confirmés » ont été choisis. F) dans la ligne, « Cas confirmés selon le test non-Trep », dans la colonne « non actif » et dans le contenu « cas confirmés » ont été sélectionnés. G) dans la rangée, les « cas confirmés selon Trep Test » ont été sélectionnés dans la colonne « non actif», dans le contenu des « cas confirmés ». H) dans la ligne sélectionnez « Cas confirmés par résidence de zone deuxième année de diagnostic », dans la colonne sélectionnez «Zone résidentielle » et dans le contenu « cas confirmés». Les données ont été compilées dans l'application Excel, un composant de la suite Microsoft Corporation Office. La recherche bibliographique a été effectuée dans des articles scientifiques, à l'aide d'ordinateurs du laboratoire informatique de l'Institut fédéral d'éducation, de science et de technologie d'Amapá, campus de Macapá, situé à: Rodovia BR 210 KM 3, s/n - Bairro Brasil Novo. CEP: 68.909-398, Macapá, Amapá, Brasil.

\section{RÉSULTATS}

$\mathrm{RC}: 68387$

Disponible en: https://www.nucleodoconhecimento.com.br/sante/femmes-enceintes 
La figure 1 montre le nombre de cas confirmés de syphilis chez les femmes enceintes par an au Brésil entre 2009 et 2013. Les données montrent une augmentation du nombre de cas confirmés de 2009 à 2012. Ce nombre a diminué de moitié en 2013 par rapport à l'année précédente.

Figure 1 Nombre de cas confirmés de syphilis chez les femmes enceintes par an au Brésil entre 2009 et 2013.

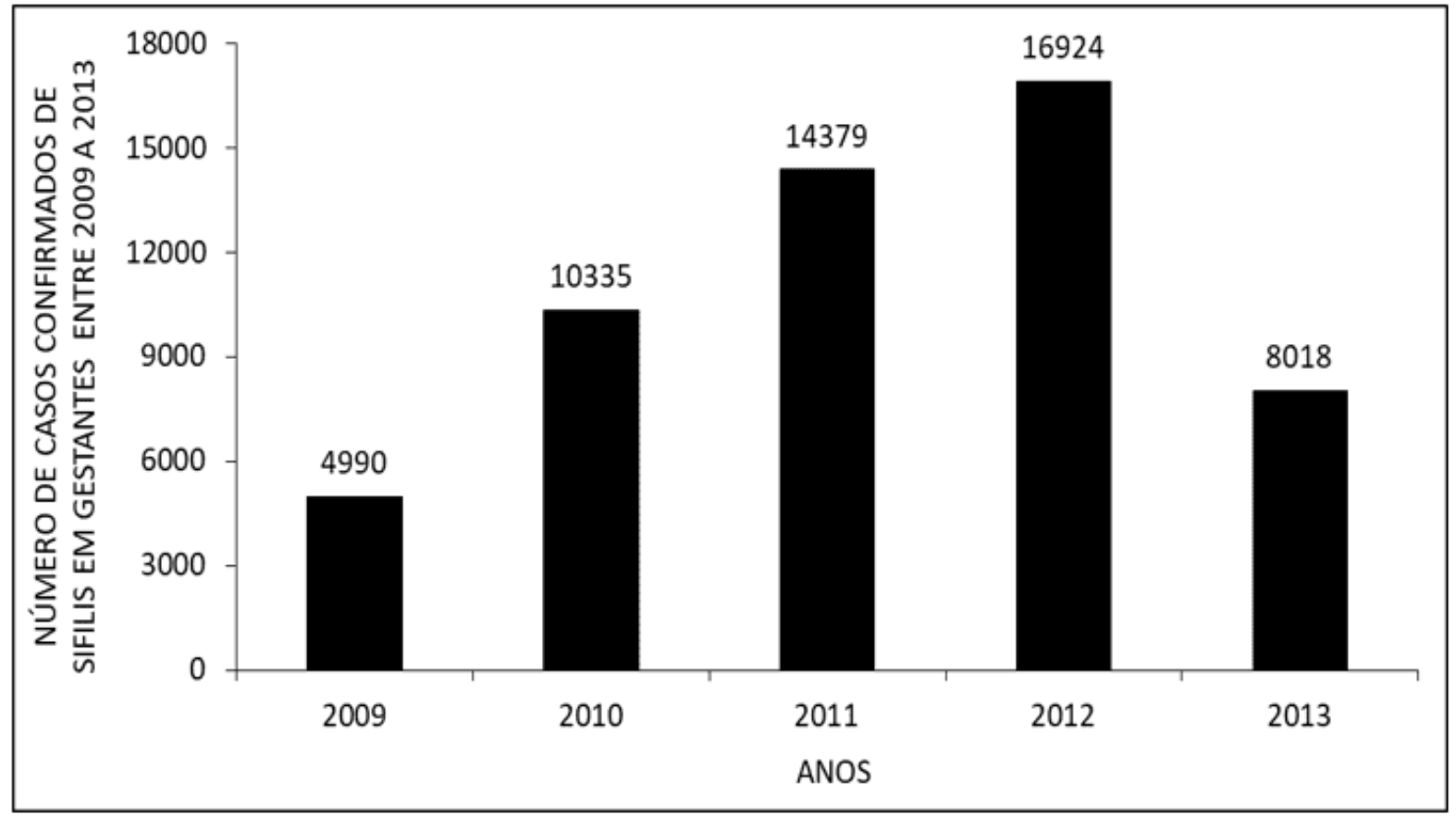

La figure 2 montre le nombre de cas confirmés de syphilis chez les femmes enceintes par groupe d'âge au Brésil entre 2009 et 2013. Entre 10 et 39 ans, il y a eu une croissance. Ce nombre diminue entre 40 et 64 ans.

RC: 68387

Disponible en: https://www.nucleodoconhecimento.com.br/sante/femmes-enceintes 
Figure 2 Nombre de cas confirmés de syphilis chez les femmes enceintes par groupe d'âge au Brésil entre 2009 et 2013.

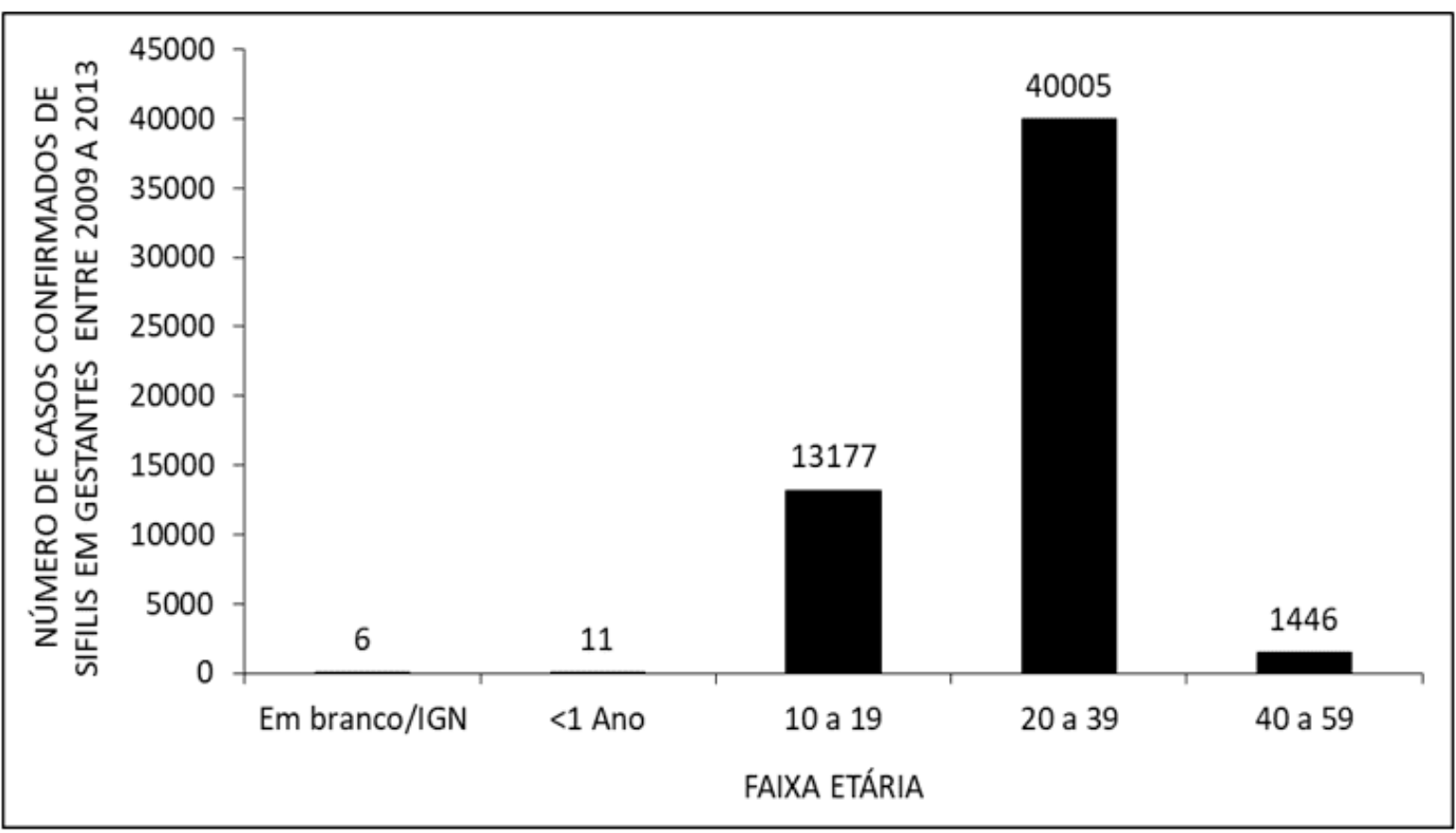

La figure 3 montre le nombre de cas confirmés de syphilis chez les femmes enceintes par région au Brésil entre 2009 et 2013. La région du sud-est a le plus grand nombre de cas confirmés, suivie respectivement par les régions du nord-est, du nord, du sud et du Midwest.

RC: 68387

Disponible en: https://www.nucleodoconhecimento.com.br/sante/femmes-enceintes 
Figure 3 Nombre de cas confirmés de syphilis chez les femmes enceintes par région du pays au Brésil entre 2009 et 2013.

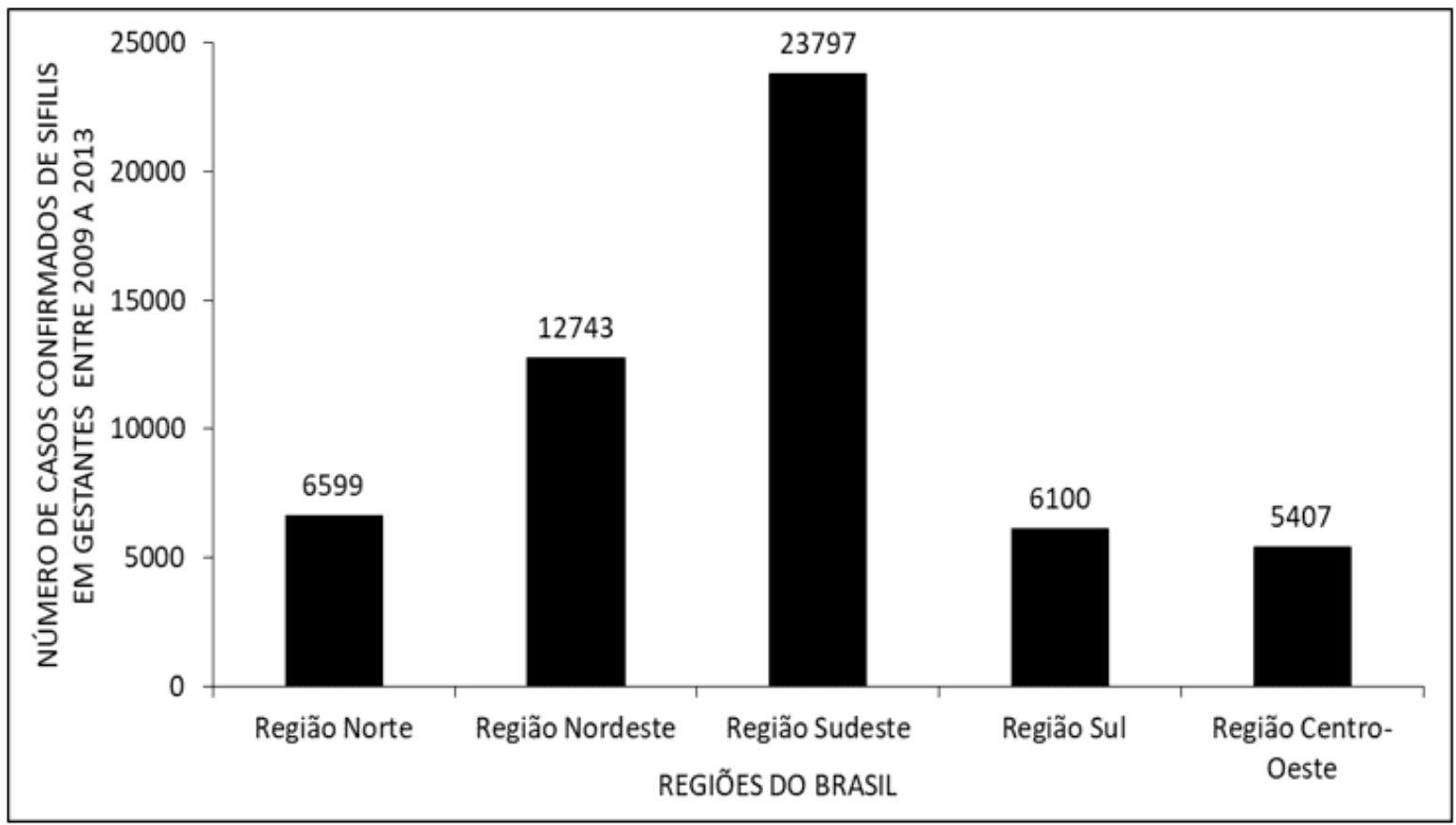

La figure 4 montre le nombre de cas confirmés de syphilis chez les femmes enceintes par zone de résidence au Brésil entre 2009 et 2013. Le nombre dans la zone urbaine est environ 8 fois plus élevé que dans la zone rurale. Le plus faible nombre de cas par zone de résidence est périurbain et Ign/Branco, respectivement.

RC: 68387

Disponible en: https://www.nucleodoconhecimento.com.br/sante/femmes-enceintes 
Figure 4 Nombre de cas confirmés de syphilis chez les femmes enceintes selon le type de zone de résidence au Brésil entre 2009 et 2013.

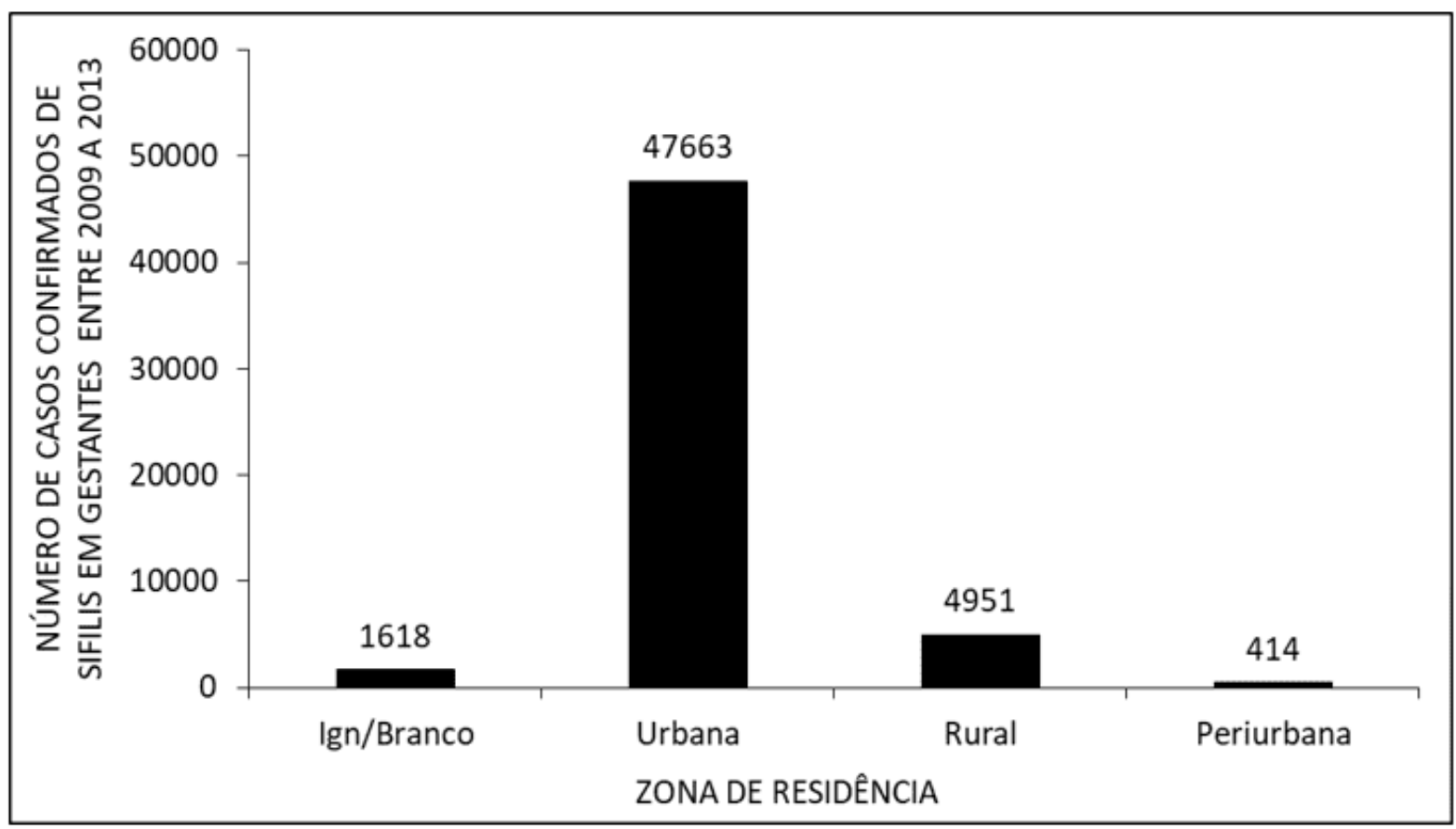

La figure 5 montre le nombre de cas confirmés de syphilis chez les femmes enceintes par type de test effectué au Brésil entre 2009 et 2013. Le nombre de cas de tests ign/blancs effectués par test TREP est supérieur aux tests NON-TREP. Le nombre de cas réactifs par des tests NON-TREP est presque deux fois plus de cas réactifs par les tests TREP. Le nombre de cas non réactifs entre les tests NONTREP et TREP est approximatif. Le nombre de cas non performants de tests TREP est neuf fois plus élevé que le nombre de cas non effectués par des tests NONTREP.

RC: 68387

Disponible en: https://www.nucleodoconhecimento.com.br/sante/femmes-enceintes 
Figure 5 Nombre de cas confirmés de syphilis chez les femmes enceintes selon le type de test effectué au Brésil entre 2009 et 2013.

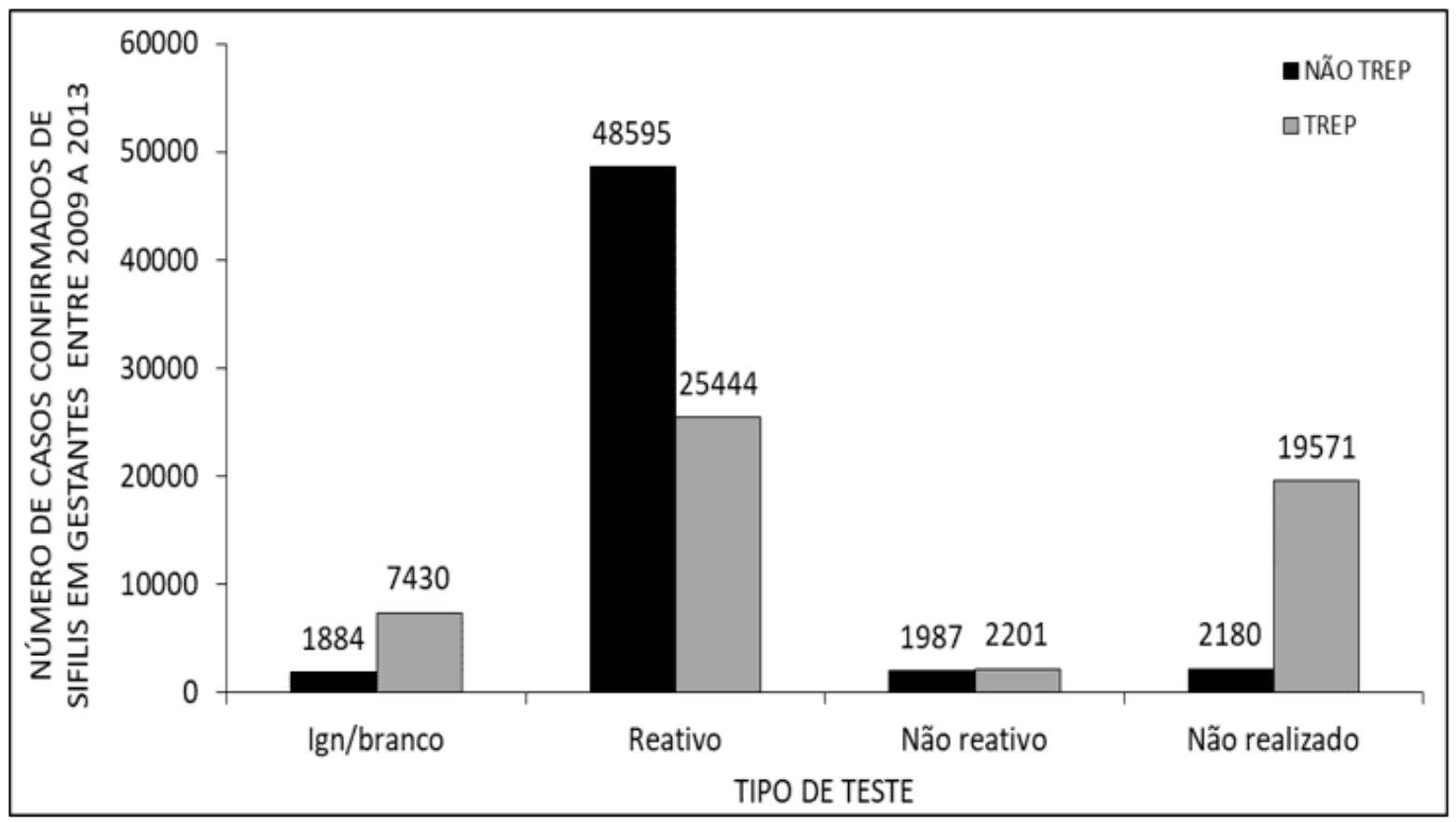

La figure 6 montre le nombre de cas confirmés de syphilis chez les femmes enceintes selon la classification au Brésil entre 2009 et 2013. La classification primaire a le plus grand nombre de cas, suivie respectivement par Ign/White et latent. Les classifications, secondaires et tertiaires, ont le plus faible nombre de cas confirmés.

RC: 68387

Disponible en: https://www.nucleodoconhecimento.com.br/sante/femmes-enceintes 
Figure 6 Nombre de cas confirmés de syphilis chez les femmes enceintes selon la classification au Brésil entre 2009 et 2013.

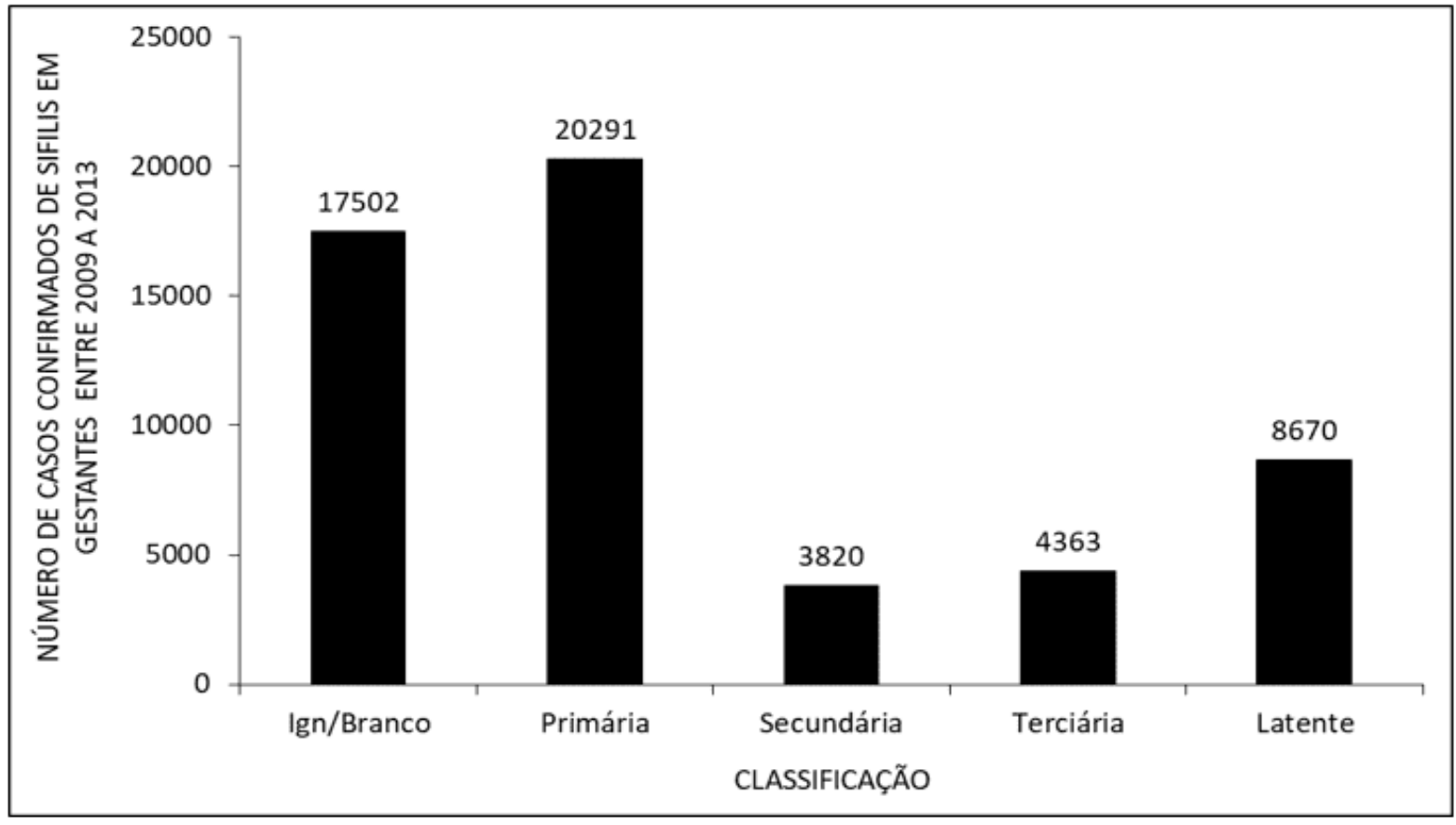

La figure 7 montre le nombre de cas confirmés de syphilis chez les femmes enceintes selon l'évolution au Brésil entre 2009 et 2013. Les données montrent que le nombre de décès de femmes enceintes qui ont contracté la syphilis au cours de cette période était deux fois plus élevé que celui des traitements. Le plus grand nombre de décès est attribuable à d'autres causes. Le plus faible nombre de décès au cours de la période a fait l'objet d'une enquête.

RC: 68387

Disponible en: https://www.nucleodoconhecimento.com.br/sante/femmes-enceintes 
Figure 7 Nombre de cas confirmés de syphilis chez les femmes enceintes selon l'évolution au Brésil entre 2009 et 2013.

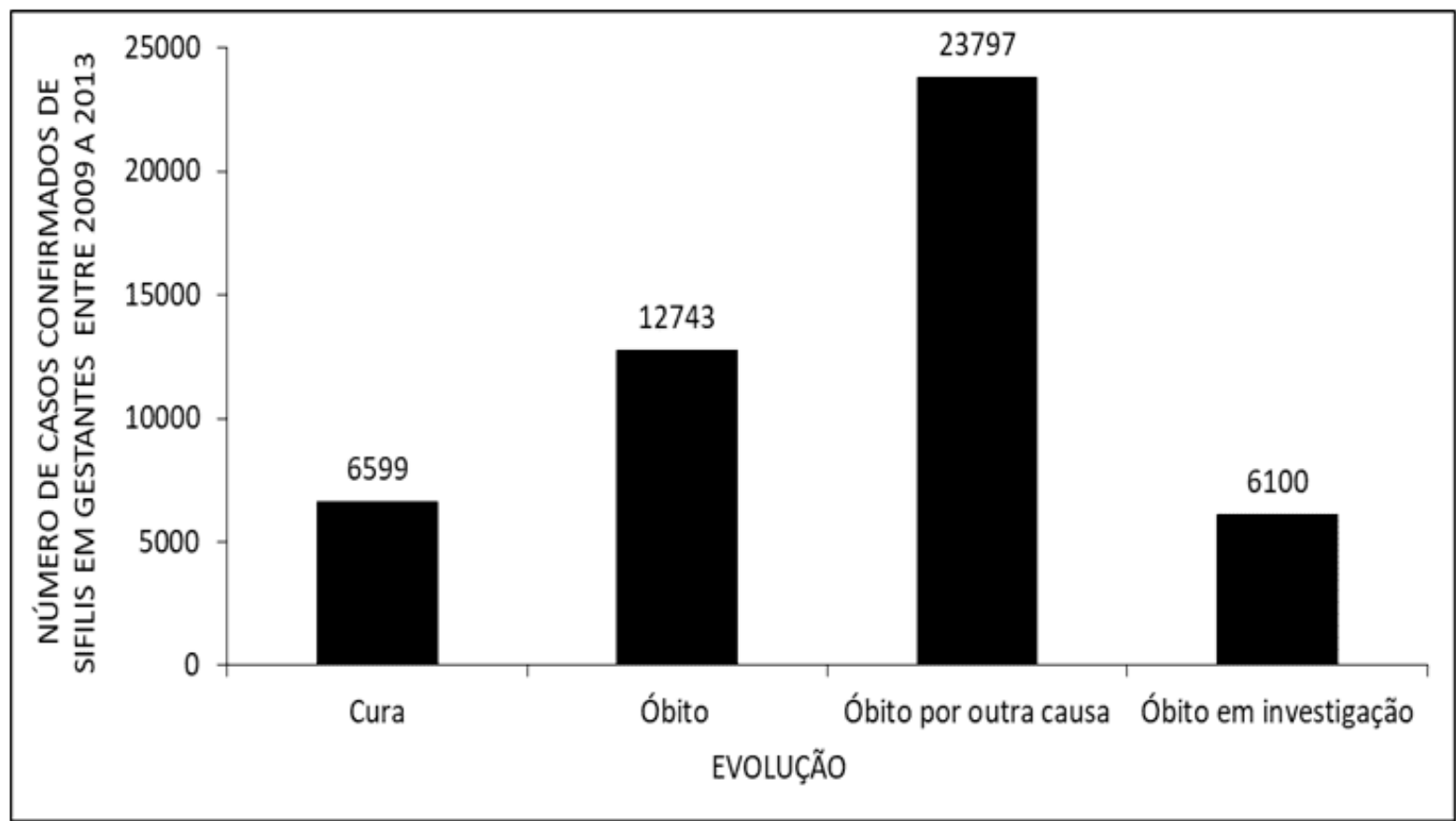

\section{DISCUSSION}

Entre 2009 et 2012, le nombre de cas confirmés de syphilis chez les femmes enceintes a augmenté. Entre 2011 et 2013, le nombre de tests a augmenté, de 31,5 mille à 1,7 million, ce qui semble avoir permis de traiter le patient atteint de syphilis. Ce scénario pourrait avoir influencé la baisse du nombre de cas confirmés chez les femmes enceintes en 2013 (BRASIL, 2013; 2017).

Les données montrent que les cas de syphilis chez les femmes enceintes ont augmenté entre 10 et 39 ans et sa diminution entre 40 et 64 ans (figure 2), corroborée par la littérature. Dans le groupe d'âge entre 20 et 34 ans, les femmes sont au sommet de leur phase de reproduction. II semble y avoir un taux plus élevé de femmes enceintes atteintes de syphilis dans ce groupe d'âge, peut-être expliqué par la pratique des rapports sexuels non protégés (COSTA et coll., 2012).

RC: 68387

Disponible en: https://www.nucleodoconhecimento.com.br/sante/femmes-enceintes 
Le Sud-Est est la région brésilienne avec le plus grand nombre de cas de syphilis chez les femmes enceintes au cours de la période étudiée. Ces données peuvent s'expliquer par le fait que le Sud-Est concentre la majorité de la population brésilienne et qu'environ la moitié de la population admet ne pas utiliser de préservatifs dans les relations sexuelles (BRASIL, 2011; IBGE, 2010).

Au cours de la période étudiée, la zone urbaine du Brésil a présenté un plus grand nombre de cas de femmes enceintes atteintes de syphilis par rapport à la zone rurale (figure 4). Selon la littérature, les gens de la population urbaine sont sexuellement liés à un plus grand nombre de partenaires. Cela peut entraîner une augmentation de la probabilité qu'une personne sans partenaire sexuel stable ou fixe contracte des infections sexuellement transmissibles comme la syphilis (BRASIL, 2011).

Le plus grand nombre de cas confirmés a été dans les tests NON-TREP que dans les tests TREP. C'est probablement parce que les tests TREP sont plus spécifiques en localisant uniquement des anticorps à la bactérie, tandis que les tests NON-TREP localiser des anticorps spécifiques et non spécifiques à la bactérie Treponema pallidum (BRASIL, 2015).

Il y avait un nombre élevé de cas confirmés de syphilis chez les femmes enceintes selon la classification primaire. On suppose qu'après le premier symptôme, dans ce cas le cancer dur, la patiente demande des soins d'hôpital, quand elle reçoit le diagnostic de syphilis primaire (AVELLEIRA et autres, 2006).

Le plus grand nombre de décès chez les femmes enceintes atteintes de syphilis était attribuable à une autre cause. Selon la littérature, plus de la moitié des décès maternels dans le monde étaient dus à l'hémorragie, à l'hypertension et à la septsémie (SAY, 2014).

RC: 68387

Disponible en: https://www.nucleodoconhecimento.com.br/sante/femmes-enceintes 


\section{CONCLUSION}

Le plus grand nombre de tests permet un meilleur traitement et une réduction du nombre de cas. Cependant, l'absence de soins préventifs pendant la relation sexuelle induit une augmentation des cas, en particulier chez les femmes en âge de procréer.

Les régions où la population est plus importante ont un plus grand nombre de cas. La forte variation des partenaires dans ces domaines entraîne une contamination accrue.

Le type de test influence les nombres. Des tests moins spécifiques et plus généraux trouvent des anticorps spécifiques et non spécifiques pour la bactérie. Cela peut modifier les nombres réels. Les soins de santé féminins font à la fois le diagnostic tôt, facilitant le traitement.

La syphilis n'est pas directement responsable du nombre plus élevé de décès chez les femmes enceintes.

\section{RÉFÉRENCES}

AVELLEITA, J. C. R.; BOTTINO, G; Sífilis: Diagnóstico, Tratamento e Controle. An. Bras. Dermatol, Vol.81 no.2 Rio de Janeiro Mar./Apr. 2006.

BRASIL São Paulo, Centro de Referência e Treinamento DST/Aids. Boletim Epidemiológico. Ano XXX. 2013.

BRASIL, Ministério da Saúde. Secretaria de Ciência, Tecnologia e Insumos Estratégicos. Relatório de Recomendação, n¹59, 2015. Disponível em: <file://C:/Users/aluno.SELABI/Desktop/CURSO\%20DE\%20ESCRITA/(BRASIL,\%20 2015).pdf $>$. Acesso em:26/09/2017.

RC: 68387

Disponible en: https://www.nucleodoconhecimento.com.br/sante/femmes-enceintes 
BRASIL. Ministério da Saúde Secretaria de Vigilância em Saúde - Departamento de DST, Aids e Hepatites Virais SAF SUL Trecho 2 Boletim Epidemiológico - Sífilis Ano IV- no 1, 2015.

BRASIL. Ministério da Saúde. Ministério da Saúde Lança Campanha de Combate à Sífilis. Disponível em: <http://www.brasil.gov.br/saude/2013/10/ministerio-dasaude-lanca-campanha-de-combate-a-sifilis>. Acesso em: 19/09/2017.

BRASIL. Ministério da Saúde. Portal da Saúde. Combate á Sífilis Congênita. Disponivel em: <http://portalarquivos.saude.gov.br/campanhas/sifilis/>. Acesso em: 19/09/2017.

BRASIL. Ministério da Saúde. Secretaria de Vigilância em Saúde. Departamento de DST, Aids e Hepatites Virais. Pesquisa de conhecimento, atitudes e práticas na população brasileira / Ministério da Saúde. Secretaria de Vigilância em Saúde. Departamento de DST, Aids e Hepatites Virais. - Brasília: Ministério da Saúde, 2011.

BRASIL. Ministério da Saúde. Secretaria de Vigilância em Saúde. Departamento de DST, Aids e Hepatites Virais. Pesquisa de conhecimento, atitudes e práticas na população brasileira / Ministério da Saúde. Secretaria de Vigilância em Saúde. Departamento de DST, Aids e Hepatites Virais. - Brasília: Ministério da Saúde, 2011.

BRETAS, J. R. S.; OHARA, C. V. S.; JARDIM, D. P.; MUROYA, R. L.; Conhecimento Sobre DST/AIDS por Estudantes Adolescentes. Rev. esc. enferm. USP vol.43 no.3 São Paulo Sept. 2009.

CARVALHO, I. S.; BRITO, R. S; Sífilis congênita no Rio Grande do Norte: estudo descritivo do período 2007-2010. Epidemiol. Serv. Saúde, v.23 n.2 Brasília, 2014.

RC: 68387

Disponible en: https://www.nucleodoconhecimento.com.br/sante/femmes-enceintes 
COSTA, C. C.; FEITAS, L. V.; SOUSA, D. M. N.; OLIVEIRA, L. L.; CHAGAS, A. C. M. A.; LOPES, M. V. O.; DAMASCENO, A. K. C. Sífilis Congênita No Ceará: Análise Epidemiológica De Uma Década. Rev Esc Enferm USP 2013; 47(1):149-56.

IBGE, Censo Demográfico 1960, 1970, 1980, 1991, 2000 e 2010. Disponível em: $<$ https://censo2010.ibge.gov.br/sinopse/index.php?dados=8>. Acesso em: 26/09/2017.

JUNIOR, W. B.; SHIRATSU, R.; PINTO, V.; Abordagem nas Doenças Sexualmente Transmissíveis. An Bras Dermatol. 2009;84(2):151-59.

KALININ, Y.; NETO, A. P.; PASSARELLI, D. H. C. Sífilis: aspectos clínicos, transmissão, Manifestações orais, diagnóstico e tratamento. Odonto 2015; 23(4546): 65-76.

MESQUITA, K.; LIMA, G. K.; FILGUEIRA, A.; FLÔR, S. M.; FREITAS, C. A.; LINHARES, M. S.; GUBERT, F.Análise dos Casos de Sífilis Congênita em Sobral, Ceará: Contribuições para Assistência Pré-Natal. DST - J bras Doenças Sex Transm 2012; 24(1):20-27

ROCHA, K.D., FECURY, A.A., OLIVEIRA, E., DENDASCK, C.V., DIAS, C.A.G.M. Number of congenital syphilis cases in Brazil between 2009 and 2013. Revista Científica Multidisciplinar Núcleo do Conhecimento. Year 05, Ed. 05, Vol. 01, pp. 131-143. May 2020.2 ISSN:2448-0959. DOI: 10.32749/nucleodoconhecimento.com.br/health/congenital-syphilis-cases.

SAY, L.; CHAU, D.; GEMMILL, A.; TUBÇALP, O.; MOLLER, A.; DANIELS, J.; GULMEZOGLU, A. M.; TEMMERMAN, M.; ALKEMA, L. Global Causes Of Maternal Death: A Who Systematic Analysis. Lancet Glob Health, Vol.2, 2014.

SUTO, C. S. S.; SILVA, D. L.; ALMEIDA, E. S.; COSTA, E. L.; EVANGELISTA, T. J. Assistência Pré-Natal A Gestante Com Diagnóstico De Sífilis Revista de Enfermagem e Atenção à Saúde 2016; 5(2): 18-33.

RC: 68387

Disponible en: https://www.nucleodoconhecimento.com.br/sante/femmes-enceintes 
Soumis : Novembre 2020.

Approuvé : Novembre 2020.

RC: 68387

Disponible en: https://www.nucleodoconhecimento.com.br/sante/femmes-enceintes 